\title{
EDITORIAL
}

\section{Nipah Virus - An Emerging zoonotic disease}

\begin{abstract}
Alebiosu C.O.
Nipah virus (NiV) is a paramyxovirus, member of the genus Henipavirus of the family Paramyxoviridae, characterized by high pathogenicity and endemic in South Asia and classified as a Biosafety Level-4 (BSL-4) agent (1). The name 'Nipah virus' originated from Sungai Nipah (Nipah River Village), where the first isolates were obtained (2-4). Bats of the genus Pteropus appear to be the natural reservoir of the virus. Nipah virus is an emerging pathogen identified in 1999 in Malaysia, Singapore, India, Bangladesh, and Philippines by Chua et al in an outbreak of acute encephalitis in pigs and humans $(5,6)$. The incubation period of Nipah has been reported to be 5 days to 2 weeks (maximum of 2 months (7). The majority of patients initially develop influenza-like signs and symptoms, including fever, headache, myalgia and vomiting.

$\mathrm{NiV}$ is an enveloped, negative-sense, single-stranded RNA virus with a large genome, consisting of over 18,000 nucleotides. The 6 genes encode for nucleocapsid $(\mathrm{N})$, phosphoprotein $(\mathrm{P})$, matrix protein (M), fusion glycoprotein $(F)$, attachment glycoprotein $(G)$ and the large polymerase. The viral $G$ protein attaches to the host cell ephrin B2 and/or B3 receptor, and activates the F protein to initiate viral envelope and host membrane fusion and viral entry (8).

Up till 2015, over 600 cases have been recorded with case fatalities between 43 and 100\% (9) at times asymptomatic, but might cause neurological and respiratory abnormalities in humans. The primary reservoir is fruit bats of the genus Pteropus while the intermediate hosts are the pigs and horses. Transmission may be from consumption of contaminated food by bats secretion or contact with infected pigs or human-to-human spread. NiV infection can be considered an emerging disease and a public health issue because of the lack of effective vaccines or therapies and the fact that NiV can infect animals such as pigs $(10,11)$. At present, there is no antiviral drug available for Nipah virus disease and the treatment is supportive.

Current prevention strategies focus on raising disease awareness in affected areas. Controlling the virus in its wild reservoir does not seem for now to be a feasible approach. However, establishing or reinforcing surveillance systems is of utmost importance to ensure that $\mathrm{NiV}$ outbreaks can be detected quickly and appropriate control measures promptly initiated. Also essential are the efforts on behavioural change communication interventions, so as to increase awareness on the risks associated with this virus.

\section{References}

1. WHO Target Product Profile for Nipah virus Vaccine, June 2017. http://www.who.int/csr/research-and-development/priority disease list review short summary 25Jan2017.pdf

2. Clayton BA. Nipah virus: transmission of a zoonotic paramyxovirus. Curr. Opin. Virol. 2017; 22:97-104. Available at: http://linkinghub.elsevier.com/retrieve/pii/S1879625716302097.

3. Marsh GA, Wang LF. Hendra and Nipah viruses: Why are they so deadly? Curr. Opin. Virol. 2012; 2:242-247. Available at: http://dx.doi.org/10.1016/j.coviro.2012.03.006

4. Chadha MS, Comer JA, Lowe L, et al. Nipah virus-associated encephalitis outbreak, Siliguri, India. Emerg. Infect. Dis. 2006; 12:235-240.

5. World Health Organization(WHO) Regional Office for South-East Asia. Nipah virus outbreaks in the WHO South-East Asia Region.

6. Luby SP. The pandemic potential of Nipah virus. Antiviral Res. 2013; 100:38-43. Available at: http://dx.doi.org/10.1016/j.antiviral.2013.07.011.

7. Goh KJ, Tan CT, Chew NK, et al. Clinical features of Nipah virus encephalitis among pig farmers in Malaysia. N Engl J Med. 2000 Apr 27;342(17):1229-35.

8. Ong KC, Wong KT. Henipavirus Encephalitis: Recent Developments and Advances. Brain Pathol. 2015 Sep;25(5):605-13.

9. Marsh GA, Wang LF. Hendra and Nipah viruses: Why are they so deadly? Curr. Opin. Virol. 2012; 2:242-247. Available at: http://dx.doi.org/10.1016/j.coviro.2012.03.006.

10. Chua KB, Bellini WJ, Rota PA, Harcourt BH, Tamin A, Lam SK, et al. Nipah virus: a recently emergent deadly paramyxovirus. Science 2000; 288: 1432-1435

11. Epstein JH, Field HE, Luby S, Pulliam JR, Daszak P. Nipah virus: impact, origins, and causes of emergence. Curr Infect Dis Rep 2006; 8(1) $59-65$.
\end{abstract}

Prof. Alebiosu C.O.

http://orcid.org/0000-0001-6224-6039

Email: olutayo.alebiosu@uniosun.edu.ng

RJHS Editor-In-Chief 


\title{
ÉDITORIAL
}

\section{Virus NIPAH, une maladie zoonotique émergente}

\begin{abstract}
Alebiosu C.O.
Le Virus NIPAH (NiV) est un paramyxovirus, appartenant au genre Hénipavirus de la famille des paramyxoviridae qui possède une forte pathogénicité et un caractère endémique en Asie du sud et est considéré comme un agent (1) biosécurité de niveau 4 (BSL-4). Le nom 'virus Nipah' vient de Sungai Nipah (village du fleuve Nipah), où les premiers isolats sont apparus (2-4). Les chauves-souris du genre Pteropus semblent être les réservoirs naturels du virus. Le virus Nipah est un agent pathogène émergent qui a été détecté pour la première fois en 1999 en Malaisie, en Singapour, en Inde, au Bangladesh et aux "Philippines par Chua et Al lors d'une épidémie d'encéphalites graves chez les porcs et les humains. La période d'incubation du Nipah est de 5 jours à 12 semaines ( 2 mois maximum (7). La majorité des patients présentent initialement des signes et symptômes de la grippe à savoir: la fièvre, les maux de tête, la myalgie et le vomissement.

Le NiV est un virus ARN enveloppé, sens négatif à simple brin avec un large génome constitué de plus de 18 000 nucléotides. Les 6 gènes codent pour le Nucléocapside $(\mathrm{N})$, la phosphoprotéine $(\mathrm{P})$, la protéine matrice $(\mathrm{M})$, la glycoprotéine de fusion $(\mathrm{F})$, la glycoprotéine de fixation $(\mathrm{G})$ et la grande polymérase. La protéine virale $\mathrm{G}$ se fixe à la cellule hôte réceptrice Ephrin B2 et/ou B3 et active la protéine F pour initier l'enveloppe virale et la membrane hôte de fusion et la pénétration virale (8).

Jusqu' en 2015 plus de 600 cas ont été signalés avec un taux de létalité de 43 à 100\% (9), parfois asymptomatiques qui cependant peuvent causer des troubles neurologiques et respiratoires chez les êtres humains. Les chauves-souris frugivores du genre pteropus sont le principal réservoir alors que les hôtes intermédiaires sont les porcs et les chevaux. La transmission viendrait de la consommation des denrées contaminées par les secrétions des chauves-souris ou du contact avec les porcs infectés ou encore d'une transmission interhumaine.

L'infection du NiV peut être considérée comme une maladie émergente et un cas qui relève de la santé publique faute de vaccins ou de traitements efficaces et à cause du fait que le virus Nipah peut aussi infecter les animaux tels que les porcs. Il n'y a pas à ce jour de médicaments antiviraux disponibles contre le virus Nipah et les traitements existants ne servent qu'à soutenir les patients. Les mesures de préventions mises sur pied s'appuient sur la sensibilisation dans les zones infectées. Le contrôle du virus dans son réservoir à l'état sauvage apparait pour l'instant comme une approche impossible. Cependant la mise en place et le renforcement de systèmes de surveillance est d'une importance capitale afin d'assurer une rapide détection et le déploiement de mesures de contrôle appropriées. Très important aussi sont les efforts de sensibilisation qui favorisent un changement de comportement afin d'éduquer les populations sur les risques liés au virus Nipah.
\end{abstract}

\section{References}

1. WHO Target Product Profile for Nipah virus Vaccine, June 2017.

http://www.who.int/csr/research-and-development/priority disease list review short summary 25Jan2017.pdf

2. Clayton BA. Nipah virus: transmission of a zoonotic paramyxovirus. Curr. Opin. Virol. 2017; 22:97-104. Available at: http://linkinghub.elsevier.com/retrieve/pii/S1879625716302097.

3. Marsh GA, Wang LF. Hendra and Nipah viruses: Why are they so deadly? Curr. Opin. Virol. 2012; 2:242-247. Available at: http://dx.doi.org/10.1016/j.coviro.2012.03.006.

4. Chadha MS, Comer JA, Lowe L, et al. Nipah virus-associated encephalitis outbreak, Siliguri, India. Emerg. Infect. Dis. 2006; 12:235-240.

5. World Health Organization(WHO) Regional Office for South-East Asia. Nipah virus outbreaks in the WHO South-East Asia Region.

6. Luby SP. The pandemic potential of Nipah virus. Antiviral Res. 2013; 100:38-43. Available at: http://dx.doi.org/10.1016/j.antiviral.2013.07.011.

7. Goh KJ, Tan CT, Chew NK, et al. Clinical features of Nipah virus encephalitis among pig farmers in Malaysia. N Engl J Med. 2000 Apr 27;342(17):1229-35.

8. Ong KC, Wong KT. Henipavirus Encephalitis: Recent Developments and Advances. Brain Pathol. 2015 Sep;25(5):605-13.

9. Marsh GA, Wang LF. Hendra and Nipah viruses: Why are they so deadly? Curr. Opin. Virol. 2012; 2:242-247. Available at: http://dx.doi.org/10.1016/j.coviro.2012.03.006.

10. Chua KB, Bellini WJ, Rota PA, Harcourt BH, Tamin A, Lam SK, et al. Nipah virus: a recently emergent deadly paramyxovirus. Science 2000 288: $1432-1435$.

11. Epstein JH, Field HE, Luby S, Pulliam JR, Daszak P. Nipah virus: impact, origins, and causes of emergence. Curr Infect Dis Rep 2006; 8(1): $59-65$.

Prof. Alebiosu C.O.

http://orcid.org/0000-0001-6224-6039

Email: olutayo.alebiosu@uniosun.edu.ng

RJHS Editor-In-Chief

Received:April 17, 2018

Accepted: May 05, 2018

Published: June 30, 2018

Research Journal of Health Sciences subscribed to terms and conditions of Open Access publication. Articles are distributed under the terms of Creative Commons Licence (CC BY-NC-ND 4.0). (http://creativecommons.org/licences/by-nc-nd/4.0).

http://dx.doi.org/10.4314/rejhs.v6i2.1 